\title{
Development of new polymyxin derivatives for multi-drug resistant Gram-negative infections
}

\author{
Pamela Brown and Michael J Dawson \\ Over the last decade, there has been a resurgence of interest in polymyxins owing to the rapid rise in multi-drug resistant \\ Gram-negative bacteria against which polymyxins offer a last-resort treatment. Although having excellent antibacterial activity, \\ the clinical utility of polymyxins is limited by toxicity, especially renal toxicity. There is much interest therefore in developing \\ polymyxin analogues with an improved therapeutic index. This review describes recent work aimed at improving the activity \\ and/or reducing the toxicity of polymyxins. Consideration to providing activity against emerging strains with reduced susceptibility \\ to polymyxins is also made.
}

The Journal of Antibiotics (2017) 70, 386-394; doi:10.1038/ja.2016.146; published online 11 January 2017

\section{INTRODUCTION}

Polymyxins are cationic cyclic lipodecapeptides which kill Gramnegative bacteria by a poorly understood sequence of events which is initiated by binding to lipopolysaccharide (LPS). Owing to their mode-of-action, polymyxins inhibit Gram-negative bacteria only, with excellent activity against important pathogens including Escherichia coli, Klebsiella pneumoniae, Pseudomonas aeruginosa and Acinetobacter baumannii. Some other species of Gram-negative bacteria including most isolates of Proteus spp., Providencia spp., Serratia spp., Brucella spp., Neisseria spp., Chromobacterium spp. and Burkholderia spp. are, however, intrinsically resistant.

First discovered in the late $1940 \mathrm{~s}^{1,2}$ and introduced into clinical practice shortly afterwards ${ }^{3}$ polymyxins fell out of favor through the 1960s-1970s due to the availability of apparently more efficacious and less toxic alternatives. However, since levels of resistance to most available classes of antibiotics have been rapidly increasing in Gram-negative bacteria, there has been a resurgence of interest in polymyxins over the last decade. ${ }^{4-6}$ Polymyxins have retained excellent activity against these multi-drug resistant (MDR) pathogens although a recent report of plasmid-mediated resistance is potentially worrisome. ${ }^{7,8}$

Two forms of polymyxin are currently in use for systemic infections: colistin methane sulfonate and polymyxin B (PMB). As colistin methane sulfonate is a prodrug, whereas $\mathrm{PMB}$ is an active entity in its own right, the properties of these two drugs are somewhat different, especially their pharmacokinetics. ${ }^{9}$ However, both drugs share the property that their dosing requires a careful compromise to achieve adequate exposure for efficacy without incurring unacceptable toxicities, particularly nephrotoxicity. ${ }^{10}$

The polymyxins were introduced into clinical practice at a time when the development pathway for antibiotics was much less detailed than currently and hence there is a dearth of information on optimum dosing for efficacy and for minimizing toxicity and resistance development, as well as a lack of basic understanding of the pharmacology and mechanisms of toxicity. The polymyxins are particularly effective in combination with other antibiotics as they appear to facilitate passage of other agents across the outer membrane of Gram-negative bacteria, but again the optimum combinations have not been established. Much work is ongoing to better characterize current polymyxins and optimize their clinical use. ${ }^{1-13}$

Although we will briefly mention some recent advances with the currently available polymyxins, the focus of this review is on the development of next generation polymyxins designed to improve therapeutic index and to provide activity against strains with reduced susceptibility to current polymyxins. The reader's attention is drawn to two previous reviews by Professor Vaara as well as a thorough compilation of earlier work by Velkov et al. ${ }^{14-16}$ We will aim to update these reviews with more recent studies.

\section{NATURAL POLYMYXINS AND RELATED NATURAL PRODUCTS} Members of the polymyxin family, isolated from strains of Paenibacillus polymyxa (previously known as Bacillus polymyxa), contain a heptapeptide core and a linear tripeptide chain, capped at the N-terminus with fatty-acyl groups of at least 7 carbon atoms. Polymyxins contain a number of cationic amino acid residues, consisting of $\alpha, \gamma$-diaminobutyric acid (Dab). The characterized components of the clinically used polymyxins B and E (Colistin) have been recently reviewed ${ }^{17,18}$ and are shown in Table 1 . A representative structure (polymyxin B1, PMB1) is shown in Figure 1. The heptapeptide core of polymyxins contains one amino acid of the D-configuration at position 6 , all the others being of the $\mathrm{L}$-configuration. The PMB family (B1-6, Table 1) all have D-phenylalanine at position 6 and constituents have been identified with variations in the fatty acyl

Cantab Anti-infectives Ltd., BioPark Hertfordshire, Welwyn Garden City, Hertfordshire, UK

This article is dedicated to Professor Julian Davies for his outstanding contributions to the antibiotic field.

Correspondence: Dr MJ Dawson, Cantab Anti-infectives Limited, BioPark Hertfordshire, Broadwater Road, Welwyn Garden City, Hertfordshire AL7 3AX, UK.

E-mail: mike.dawson@cantabanti.com

Received 12 September 2016; revised 2 November 2016; accepted 12 November 2016; published online 11 January 2017 
Table 1 Naturally occurring polymyxins. All amino acids refer to the L-configuration unless otherwise specified

\begin{tabular}{lllllll}
\hline Name & Fatty-acyl tail & AA-2 & AA-3 & AA-6 & AA-7 & AA-10 \\
\hline PMB1 & 6-MOA & Thr & Dab & D-Phe & Leu & Thr \\
Ile-B1 & 6-MOA & Thr & Dab & D-Phe & isoLeu & Thr \\
B2 & 6-MHA & Thr & Dab & D-Phe & Leu & Thr \\
B3 & Octanoic acid & Thr & Dab & D-Phe & Leu & Thr \\
B4 & Heptanoic acid & Thr & Dab & D-Phe & Leu & Thr \\
B5 & Nonanoic acid & Thr & Dab & D-Phe & Leu & Thr \\
B6 & 3-hydroxy-6-methyl & Thr & Dab & D-Phe & Leu & Thr \\
& octanoic acid & & & & & \\
Polymyxin E1 & 6-MOA & Thr & Dab & D-Leu & Leu & Thr \\
E2 & 6-MHA & Thr & Dab & D-Leu & Leu & Thr \\
E3 & Octanoic acid & Thr & Dab & D-Leu & Leu & Thr \\
E4 & Heptanoic acid & Thr & Dab & D-Leu & Leu & Thr \\
& & & & & & \\
E1-ileu (circulin) & 6-MOA & Thr & Dab & D-Leu & isoLeu & Thr \\
E1-val & 6-MOA & Thr & Dab & D-Leu & Val & Thr \\
E1-NVa & 6-MOA & Thr & Dab & D-Leu & norVal & Thr \\
E2-ile & 6-MHA & Thr & Dab & D-Leu & isoLeu & Thr \\
E2-val & 6-MHA & Thr & Dab & D-Leu & Val & Thr \\
E8-ile & 7-methyl nonanoyl & Thr & Dab & D-Leu & isoLeu & Thr \\
E1-(ser-2) & 6-MOA & Ser & Dab & D-Leu & Leu & Thr \\
Polymyxin D1 & 6-MOA & Thr & D-Ser & D-Leu & Thr & Thr \\
Polymyxin S1 & 6-MOA & Thr & D-Ser & D-Phe & Thr & Thr \\
Polymyxin M & 6-MOA & Thr & Dab & D-Leu & Thr & Thr \\
Polymyxin A & 6-MOA & Thr & D-Dab & D-Leu & Thr & Thr \\
PMB1 (D-Dab-3) & 6-MOA) & Thr & D-Dab & D-Phe & Leu & Thr \\
PMB2 (D-Dab-3) & 6-MHA) & Thr & D-Dab & D-Phe & Leu & Thr \\
Polymyxin T & 6-MOA & Thr & Dab & D-Phe & Leu & Leu
\end{tabular}

Abbreviations: 6-MOA, 6-methyl octanoic acid; 6-MHA, 6-methyl heptanoic acid; AA, amino acid; Dab, diaminobutyric acid; Leu, Leucine; Phe, phenylalanine; PMB, polymyxin B; Ser, serine; Thr, threonine.

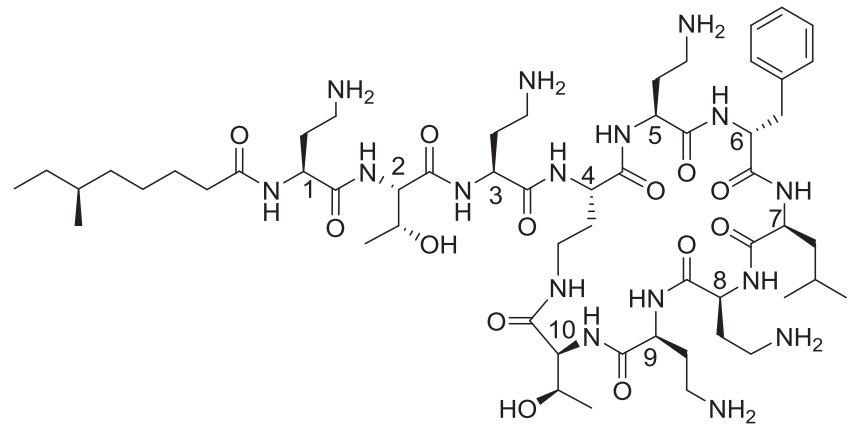

Figure 1 Structure of polymyxin B1 (PMB1) showing amino acid numbering.

chain, ${ }^{19}$ as well as replacement of leucine with isoleucine at position 7 (Ile-B1, Table 1). Polymyxin E components are characterized by D-Leu at position 6. At position 7, in place of L-leucine a number of variants have also been reported including the isoleucine-7 variant, circulin, as well as valine or norvaline. A variant in which threonine-2 has been replaced by serine has also been reported. ${ }^{20}$ Polymyxin D and Polymyxin S are structurally related, ${ }^{21}$ both having threonine at position 7 and D-Serine at position 3. Polymyxin M (Mattacin), ${ }^{22}$ produced by the soil bacterium Paenibacillus kobensis $\mathrm{M}$ also has threonine at position 7, in conjunction with Leu-6. Threonine-7 is also present in Polymyxin A, which, unusually, possesses the D-Dab residue at position 3. Although first reported in $1947,{ }^{1}$ no further examples of this were isolated until 2011, when the D-Dab forms of PMB1 and B2 were found to be produced by P. polymyxa PKB-1. ${ }^{23} \mathrm{~A}$ variant in which Thr-10 has been replaced by Leucine (Polymyxin T) has also been characterized. ${ }^{24}$

A family of related natural products called the octapeptins ${ }^{25}$ was first isolated in the 1970s. Naturally occurring variants which have been reported in the literature are shown in Table 2. In common with the polymyxins, the octapeptins also possess a heptapeptide core rich in Dab residues, but with only one exocyclic amino acid. As with the Polymyxins, the heptapeptide core contains one amino acid of the D-configuration. All octapeptins have a hydroxylated fatty acyl chain on the N-terminus. Figure 2 shows Octapeptin A1, first isolated as EM49- $\beta$, which contains D-leucine at position 4 and leucine at position 5..$^{25,26}$ The Octapeptins B1-3 are similar to the A family, but have a phenylalanine residue at position $5 .^{27}$ The Octapeptin C series, which was first isolated as $\mathrm{Bu}-2470$ contains a D-phenylalanine residue at position $4,{ }^{28,29}$ and related compounds have also been isolated in this series. Most of the octapeptins possess the amino acid $\mathrm{D}-\mathrm{Dab}$ at the position adjacent to the heptapeptide core. However, Octapeptin D has D-Serine as the exocyclic amino acid. ${ }^{30}$ A related octapeptide, Battacin, Octapeptin B5 was isolated in 2012.31 This is reported to have the D-amino acid at position 5 of the cyclic core, and this was recently confirmed by total synthesis. ${ }^{32}$

\section{MODE-OF-ACTION}

The interaction of polymyxins with LPS has been studied at the molecular level. ${ }^{33}$ However, the sequence of events that then leads to cell-death, for these rapidly cidal antibiotics, is poorly understood. It is hypothesized that the initial interaction with LPS is facilitated by electrostatic interaction between the positively charged Dab residues of polymyxin with the negatively charged phosphate groups and carboxylates on LPS. Destablisation of LPS, perhaps involving displacement of calcium ions, facilitates uptake of polymyxin and interaction with the inner membrane. It has long been known that polymyxins also facilitate uptake of hydrophobic antibiotics making them excellent partners in combination regimens. ${ }^{34}$ Recent work using newly developed probes supports the internalization of polymyxins in a similar time-frame to their bactericidal activity. ${ }^{35}$

\section{TOXICITY}

Polymyxins express a range of toxicities in mammalian systems including histamine-release, neurotoxicity and nephrotoxicity. ${ }^{36,37}$ The nephrotoxicity is dose-limiting necessitating a careful balance between the dose required for efficacy and that which causes an unacceptable level of toxicity. ${ }^{10}$ Colistin has been developed in a prodrug form (colistin methane sulfonate) to reduce toxicity, but PMB is dosed in the active form. There is no consensus on the relative toxicity of the two drugs in clinical use although some recent studies appear to favor PMB. ${ }^{38,39}$ Undoubtedly PMB, as an active drug moiety in its own right, has simpler pharmacokinetics than the prodrug colistin methane sulfonate which suffers rapid renal clearance and variable cleavage to the active form. ${ }^{9}$ Reduction of nephrotoxicity is a key target for improvement of the polymyxin class. Most efforts to develop new polymyxins are not following a prodrug approach.

The mechanism of toxicity has been studied but not fully defined. Polymyxins are extensively reabsorbed after renal filtration and accumulate to high levels in the kidney where they appear to cause both apoptotic and necrotic damage to kidney cells. ${ }^{40}$ Polymyxins demonstrate binding to the brush border membrane of the renal cortex as measured by the displacement of radiolabelled gentamicin. ${ }^{41}$ A range of immortalized cell lines derived from kidney proximal 
Table 2 Structural variation in naturally occuring octapeptins. Unless specified, amino acids (AA) refer to the L-stereochemistry

\begin{tabular}{lllll}
\hline Name & Fatty acyl tail & $A A-1$ & $A A-4$ & $A A-5$ \\
\hline Octapeptin A1 & 3-hydroxy-8-methyl decanoic acid & D-Dab & D-Leu & Leu \\
Octapeptin A2 & 3-hydroxy-8-methyl nonanoic acid & D-Dab & D-Leu & Leu \\
Octapeptin A3 & 3-hydroxy- $n$ - decanoic acid & D-Dab & D-Leu & Leu \\
Octapeptin B1 & 3-hydroxy-8-methyl decanoic acid & D-Dab & D-Leu & Phe \\
Octapeptin B2 & 3-hydroxy-8-methyl nonanoic acid & D-Dab & D-Leu & Phe \\
Octapeptin B3 & 3-hydroxy-n- decanoic acid & D-Dab & D-Leu & Phe \\
Octapeptin C1 & 3-hydroxy-6-methyl octanoic acid & D-Dab & D-Phe & Leu \\
Octapeptin B5 & 3-hydroxy-6-methyl octanoic acid & D-Dab & Leu & D-Phe \\
(Battacin) & & & & \\
Octapeptin D1-3 & As Octapeptin A1-3 & D-Ser & D-Leu & Leu \\
\hline
\end{tabular}

Abbreviations: Dab, diaminobutyric acid; Leu, Leucine; Phe, phenylalanine.<smiles>[Y7]C(NC(=O)[C@H](CCN)NC(=O)[C@H](CC(C)C)NC(=O)[C@H](CC(C)C)NC(=O)[C@H](CCN)NC(=O)[C@H](CCN)NC(=O)[C@H](CCN)NC(=O)[C@H](CCN)NC(=O)[C@H](CCN)NC(=O)CC(O)CCCCC(C)CC)C(=O)N[C@@H](CCN)CC(C)C</smiles>

Figure 2 Structure of Octapeptin A1 showing amino acid numbering.

tubule cells (eg HK-2, LLC-PK1) has been used to compare analogues, although primary cultures may be more predictive. ${ }^{42}$

\section{RESISTANCE}

Resistance mechanisms to polymyxins have been reviewed recently ${ }^{43}$ and only brief comment will be made here. As noted above polymyxins interact with LPS and the major mechanisms of resistance involve the remodeling of LPS to reduce this interaction. Reduction of the negative charge on LPS by conjugation with positively charged moieties, predominantly 4-amino-4-deoxy-L-arabinose or phosphoethanolamine, reduces interaction with the polymyxin amines. Resistance is primarily adaptive and regulated by multiple two-component systems which can be triggered by environmental stimuli. Stable resistant mutants often involve mutation of these two component systems, however, recently plasmid-mediated acquired resistance has been identified through a transmissable phosphoethanolamine transferase denoted MCR-1. ${ }^{7}$ Activity against resistant strains presents a further goal for development of second generation polymyxins.

\section{SYNTHESIS OF POLYMYXIN DERIVATIVES}

Novel synthetic variants of polymyxin have been prepared utilizing either a total synthesis approach via solid-phase peptide synthesis (SPPS), allowing modification at points in the core heptapeptide of polymyxin, or through semi-synthetic modification via a suitable protection and deprotection strategy, allowing modification of the linear tripeptide and/or the N-terminus.

Three key methods have been used for SPPS:

(i) Linear peptide synthesis followed by off-resin cyclisation. This allows modifications at all core positions apart from threonine-10, which is used as the resin attachment point. The first approach, in $1999^{44}$ utilized 9-fluorenylmethoxycarbonyl (FMOC)- $\mathrm{Thr}\left(\mathrm{t}^{\mathrm{Bu}}\right)$ SASRIN resin. The side chain amines of the Dab residues were protected with tert-butyloxycarbonyl (BOC), with orthogonal protection (Dde) at the $\mathrm{N}^{\gamma}$ of Dab-4. The linear peptide was synthesized using conventional FMOC methodology, and the Dde protecting group removed with hydrazine, followed by acidic cleavage from the resin. The Thr-10 to Dab-4 cyclisation was effected off-resin using diphenylphosphoryl azide, followed by deprotection to afford the synthetic polymyxin in $20 \%$ yield. This approach has since been followed by several groups, each attempting to overcome some of the drawbacks, including the slow cyclisation step, and the careful cleavage from resin to avoid concomitant deprotection. A similar approach using TCP resin and iv(Dde) orthogonal protection has been used by Velkov et al. ${ }^{45}$ Vaara et $a l,{ }^{41}$ used benzyloxycarbonyl (CBZ) protection on the Dab groups allowing facile acid-induced cleavage from the resin, while Kanazawa ${ }^{46}$ utilized 2-Chloro CBZ protection which required final removal with HF. A recent publication by Ramesh et al 47 reports a clean cyclisation reaction off-resin using PyAOP (7-azabenzotriazol-1-yloxy)tripyrroldinophosphonium hexafluorophosphate) as coupling agent.

The use of the Kenner 'safety-catch' approach in which release from the resin was effected by the cyclisation step was investigated by De Visser et al. ${ }^{48}$ Although promising, the low yield of the required sulfonamide-linked threonine may be the reason why this approach has not been developed further.

(ii) Branched peptide synthesis followed by off-resin cyclisation. This approach avoids the use of protected threonine in the cyclisation step, allowing for modification at position 10 . Kline et a ${ }^{20}$ reported that a branched approach in which leucine-7 was tethered to 2-chlorotrityl resin resulted in fewer side-products than the linear approach described in (i). The disadvantage was that modification of the 7-position could not be investigated by this approach. However, for modification of other positions, or for the synthesis of a scaffold for N-terminal modification, this approach has been successfully utilized. In particular Pfizer developed this approach for the synthesis of $100 \mathrm{~g}$ of a protected Polymyxin decapeptide template. ${ }^{49}$

(iii) On-resin cyclisation. Two recent reports have described the versatility of on-resin cyclisation, avoiding any problems associated with deprotection on removal from the resin.

In the approach of $\mathrm{Xu}$ et al ${ }^{50}$ the $\gamma$-amino group of Dab- 9 was tethered (as its allyl ester) to 2-chlorotrityl (CTC) resin followed by an orthogonal protection strategy to afford the branched peptide with thr-10 as the N-terminus. Deprotection of the ester enabled cyclisation between the carboxylic acid of Dab-9 and the amine of threonine-10 on-resin using PyAOP as coupling agent. Although the reported yield of $25 \%$ overall is modest, the convenience of being able to synthesize on-resin means that this methodology deserves more attention.

At the same time, the Cooper group at the University of Queensland $^{51}$ reported an on-resin cyclisation method in which the hydroxyl group of FMOC-threonine $O$-allyl ester (to become threonine-10 in the final product) was tethered onto 3,4-dihydropyran-2-yl-methoxymethyl polystyrene resin. This enabled solid-phase peptide synthesis to proceed via the linear approach via standard FMOC chemistry using BOC protection of the Dab groups. Use of the alloc protecting group for the Dab-4 side chain amine 
enabled deprotection to take place at the same time as the threonine allyl ester, and cyclisation was effected on-resin by treatment with diphenylphosphoryl azide.

Semi-synthetic approaches offer little opportunity to modify the residues of the cyclic core of the polymyxin molecule, although one report of functionalisation at the $\mathrm{N}^{\gamma}$ of Dab- 9 has been claimed by selective BOC protection of the Dab groups at positions 1, 3, 5 and 8 of Polymyxin monohydrochloride. ${ }^{52}$ However, the majority of semisynthetic approaches entail protection of the Dab residues followed by peptide cleavage and lead to modification of the linear tripeptide and N-terminus.

Enzymic cleavage offers an efficient approach to the preparation of un-protected deacylated polymyxins. However, for synthetic utility, this approach requires either a water-soluble protecting group, for compatibility with the enzyme, or the use of an enzyme compatible with organic solvents.

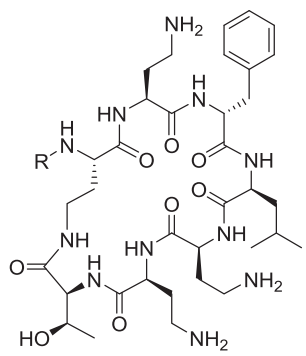

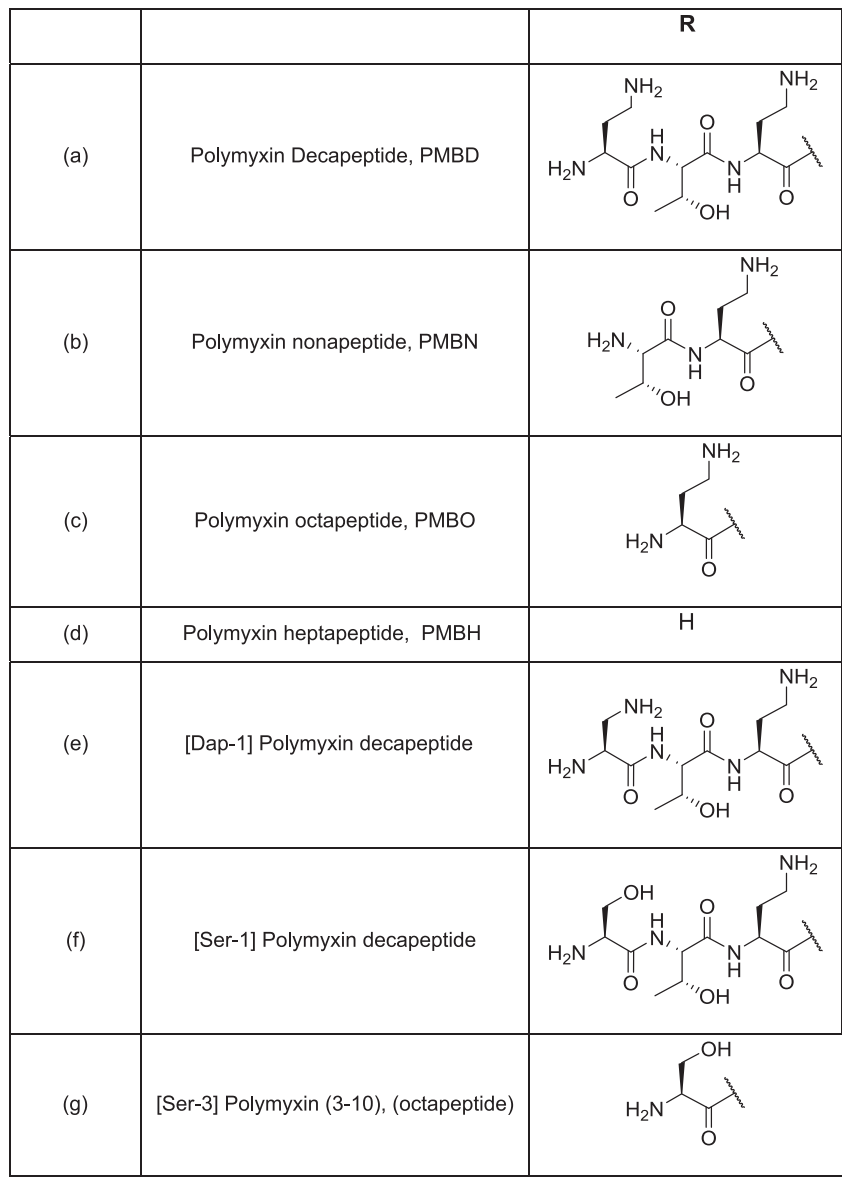

Figure 3 Des-fatty acyl polymyxins.
Polymyxin can be hydrolyzed to the deacylated polymyxin decapeptide (PMBD, Figure 3a) with polymyxin acylase, or to the polymyxin nonapeptide PMB (2-10) (PMBN; 3b) with papain. Treatment with bromelain leads to the polymyxin octapeptide PMB (3-10) (PMBO; 3c) or with Nagarse (Subtilisin B, Savinase) to the polymyxin heptapeptide $\mathrm{PMB}(4-10)(\mathrm{PMBH} ; 3 \mathrm{~d}) .{ }^{53}$ A chemical approach using Troc protection followed by cleavage with methane sulfonic acid leading to the protected PMBN has been employed by Okimura et al..$^{54}$, albeit in low yield.

Selective BOC-protection of the $\mathrm{N}^{\gamma}$ of Dab residues of PMBN in the presence of the alpha-amino group has been reported to give the tetra-BOC nonapeptide with the $\mathrm{N}$-terminal $\mathrm{N}^{\alpha}$ free for further derivatisation. ${ }^{55}$

Polymyxin decapeptide in which the Dab- $\mathrm{N}^{\gamma}$ amines are protected has been described by Cubist ${ }^{56}$ who used the water-soluble protecting group sulfonyl FMOC to enable enzymic cleavage in an aqueous medium. Protection of the Dab residues of Polymyxin with this group, followed by cleavage with Polymyxin acylase afforded the suitably protected PMB decapeptide which was used in the synthesis of a series of derivatives at the $\mathrm{N}$-terminus.

Cleavage of the fully BOC-protected polymyxin to the protected heptapeptide $\mathrm{PMBH}$ has been reported. An early patent ${ }^{57}$ described the use of the protease Nagarse (subtilisin B) in a mixture of water and 1,4butanediol to effect this cleavage. More recently, Pfizer described the same transformation ${ }^{58}$ in an acetonitrile/water mixture in good overall yield, followed by coupling to an acylated tripeptide to form a polymyxin decapaptide analog. This methodology has been further developed by Cantab Anti-infectives, ${ }^{59}$ who re-introduced a dipeptide moiety to achieve nonapeptide building blocks for further modification.

\section{DEVELOPMENT OF NEW POLYMYXINS}

The early development of the structure-activity relationship (SAR) of polymyxins has been well described by Velkov et al. ${ }^{16}$ A key early finding of Vaara ${ }^{34,60}$ was that PMB (2-10) (PMB nonapeptide 'PMBN'; 3b) retained the ability to permeabilise the outer membrane to ingress of dyes or hydrophobic antibiotics whilst having much reduced antibacterial activity. Further, this molecule had much reduced toxicity leading to a crude model that amino acids $2-10$ are involved in LPS binding and permeabilisation whereas the N-terminal fatty-acyl moiety is crucial for bacterial killing, but also contributes significantly to the toxicity of the molecule against mammalian systems. Further the cyclic core alone (PMB 4-10; PMB heptapeptide $3 \mathrm{~d})$ retains substantial ability to permeabilise the outer membrane. ${ }^{53}$ Kanazawa et al. ${ }^{46}$ further investigated the roles of the individual amino acids of Polymyxin by alanine scanning and confirmed the importance of the diaminobutyrate (Dab) residues in the cyclic core as would be expected from the model of Mares et al. ${ }^{33}$ These early findings have guided the design of next generation polymyxins.

\section{DERIVATIVES WITHOUT THE N-TERMINAL ACYL CHAIN}

Given the early findings of the reduced toxicity of PMBN, removal of the acyl chain has been of interest for reduction of toxicity of this class. There has been considerable interest in desacyl polymyxins for use in combination with other antibiotics. Indeed the alarming emergence of multi-drug resistant Gram-negative bacteria has engendered significant interest in antibiotic combinations as indeed is already a common approach in treatment of tuberculosis and many viral infections.

\section{PMBN}

PMBN itself has not thus far attracted interest for drug development perhaps due to uncertainty as to just how much its renal toxicity is 
reduced compared with $\mathrm{PMB}$ and Colistin, as well as to the regulatory challenges of developing antibiotic combinations. A recent study by Kierstead et al. ${ }^{61}$ using urinary kidney injury biomarkers to predict nephrotoxicity in a rat model has thrown further light on PMBN toxicity. Encouragingly PMBN showed a $\mathrm{TC}_{50}$ against the immortalized human renal proximal tubule cell line HK-2 of $>1000 \mu \mathrm{M}$ compared with $20 \mu \mathrm{M}$ for PMB. Dosing PMBN 4 times per day subcutaneously at $40 \mathrm{mg} \mathrm{kg}^{-1}$ per day for 7 days (plasma exposure $186 \pm 27 \mu \mathrm{g} \mathrm{h} \mathrm{ml}^{-1}$ ) resulted in no histological changes in the kidney compared with a vehicle control. Neither was there any significant elevation in blood urea nitrogen or plasma creatinine which could have indicated impaired kidney function. On the other hand Colistin dosed at the lower dose of $25 \mathrm{mg} \mathrm{kg}^{-1}$ per day $\left(92 \pm 31 \mu \mathrm{g} \mathrm{h} \mathrm{ml}^{-1}\right)$ resulted in tubular degeneration with increased vacuolization of the cytoplasm, tubular basophilia, occasional necrotic cells or casts in the tubular lumina and increased mitotic figures, although changes were considered minimal to slight. Nevertheless reduced kidney function was witnessed by modest but significant increases in blood urea nitrogen and plasma creatinine. Urinary biomarkers proved a more sensitive measure of the mild kidney damage with KIM- 1 and $\alpha$-GST showing the best predictive sensitivity. Neither of these biomarkers was significantly elevated in PMBN-treated rats.

\section{NAB741}

The Vaara group, under the corporate identity of Northern Antibiotics Ltd., have extended these studies on low toxicity antibiotic potentiators to molecules carrying only three positive charges. ${ }^{62}$ Most notably NAB741 (Figure 4a) has the identical cyclic core to PMB but with an $\mathrm{N}$-acetyl-threonine-D-serine side chain. It thus only has three positive charges compared with the five positive charges of PMB or PMBN which the Vaara group postulates influences the renal handling and hence possibly toxicity of these compounds.

NAB741 has little intrinsic antibacterial activity, but retains the capability to potentiate the activity of rifampin, clarithromycin, azithromycin and even narrow spectrum agents such as mupirocin, fusidic acid and vancomycin against $E$. coli, $K$. pneumoniae and A. baumannii but not $P$. aeruginosa. The loss of potentiating activity against $P$. aeruginosa is presumably due to the substitution of D-serine for Dab at amino acid 3 as $N$-acetyl-PMBN retains some direct activity against $P$. aeruginosa. ${ }^{63}$ Interestingly, the renal clearance of NAB741 in rats was 4000-fold higher than that of colistin.

Recently NAB741 has entered preclinical development with Spero Therapeutics as SPR741. Most encouragingly SPR741 was not associated with any nephrotoxicity when dosed up to $60 \mathrm{mg} \mathrm{kg}^{-1}$

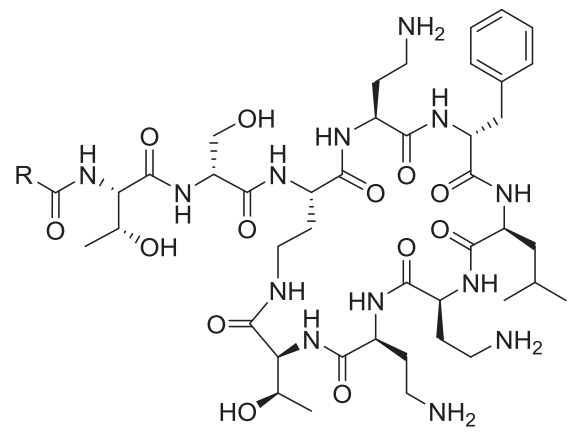

(a) NAB-741 R=CH

(b) NAB-739 R=CH$\left(\mathrm{CH}_{2}\right)_{6}-$

Figure 4 Northern antibiotics nonapeptides. per day in Cynomolgus monkeys whereas PMB demonstrated the anticipated nephrotoxicity at a dose of $12 \mathrm{mg} \mathrm{kg}^{-1}$ per day. ${ }^{64}$

\section{DES-FATTY ACYL POLYMYXIN B ANALOGUES}

Interestingly des-fatty acyl PMB (PMBD, 3a) retains moderate direct antibacterial activity especially against Pseudomonas aeruginosa. Katsuma et al. ${ }^{65}$ prepared a series of PMBD analogues where the $\mathrm{N}$-terminal amino acid was substituted by different natural amino acids. Promisingly either the diaminopropionate (Dap; 3e) or serine (3f) analogues had activity against $P$. aeruginosa similar to that of PMB, although activity against E. coli or Salmonella typhimurium was much decreased. The Dap analog had reduced acute toxicity in mouse with an $\mathrm{LD}_{50}$ of $23.5 \mu \mathrm{mol} \mathrm{kg}{ }^{-1}$ compared with $4.8 \mu \mathrm{mol} \mathrm{kg}^{-1}$ for PMB. In a subsequent study, Sato et al. ${ }^{66}$ described further des-fatty acyl nonapeptides and even octapeptides. $\left[\mathrm{Ser}^{3}\right]-\mathrm{PMB}(3-10)(3 \mathrm{~g})$ was highlighted with a minimum inhibitory concentration (MIC) against $P$. aeruginosa of $2 \mu \mathrm{g} \mathrm{ml}^{-1}$ compared with $1 \mu \mathrm{g} \mathrm{ml}^{-1}$ for PMB and an $\mathrm{LD}_{50}$ of $>50 \mu \mathrm{mol} \mathrm{kg}^{-1}$. These compounds are considered Pseudomonas-specific antibiotics. The renal handling or specific renal toxicity of these compounds has not been reported.

\section{DECAPEPTIDE DERIVATIVES WITH AN ALTERED N-TERMINAL ACYL CHAIN}

Given the strong contribution of the N-terminal acyl chain to toxicity, but also activity, a further approach to improvement of polymyxins has been to attempt to replace the acyl chain with a moiety which improves the ratio of activity to toxicity. Earlier work in this area is reviewed by Velkov et al. ${ }^{16}$ As well as exploring acyl chain length, a wide range of aryl or cycloalkyl derivatives has been investigated and many derivatives retained good potency. There has, however, been no systematic evaluation of the effect on toxicity, particularly renal toxicity.

More recently two companies have explored derivatives in this area in further detail.

\section{CB-182 804}

In collaboration with BioSource Pharm, Cubist Pharmaceuticals disclosed a range of polymyxin decapeptide derivatives with haloaryl moieties at the N-terminus. ${ }^{56}$ A urea linked 2-chlorophenyl derivative, CB-182 804 (Figure 5) was selected for further development. The activity of CB-182 804 was explored against a wide range of clinical isolates and this compound was shown to have $\mathrm{MIC}_{50}$ and $\mathrm{MIC}_{90}$ values approximately one dilution $(2 \times)$ less potent than PMB. ${ }^{67}$ Efficacy was determined in thigh and lung infection models and was generally similar to $\mathrm{PMB}$ and colistin. ${ }^{68}$

CB-182 804 had an $\mathrm{EC}_{50}$ against the rat renal proximal tubule cell line LLC-PK1 of $>1000 \mu \mathrm{g} \mathrm{ml}^{-1}$ compared with $318 \mu \mathrm{g} \mathrm{ml}^{-1}$ for PMB. Nephrotoxicity was assessed in the Cynomolgus monkey. ${ }^{69}$ No significant histopathological changes were noted at doses up to $3.2 \mathrm{mg} \mathrm{kg}^{-1}$ bid whereas the same dose tid resulted in significant lesions comparable with PMB dosed at $3.8 \mathrm{mg} \mathrm{kg}^{-1}$ bid. This may have indicated some advantage, but the plasma exposure of PMB after a $3.8 \mathrm{mg} \mathrm{kg}^{-1}$ dose was significantly higher than that for CB-182,804 after a $3.2 \mathrm{mg} \mathrm{kg}^{-1}$ dose $\left(\mathrm{AUC}_{\text {inf }} 61-74 \mu \mathrm{g} \mathrm{h} \mathrm{ml}{ }^{-1}\right.$ compared with $27-28 \mu \mathrm{g} \mathrm{h} \mathrm{m}^{-1}$ ). CB-182 804 was advanced into a phase I clinical trial but the results have not been published and the compound does not appear to have been progressed further.

\section{PFIZER $5 \times$}

Magee et al. ${ }^{49}$ reported a series of analogues in which the fatty acyl terminus of $\mathrm{PMB}$ was replaced by a variety of biaryl moieties. 
<smiles>CC(C)C[C@H](NC(=O)[C@H](Cc1ccccc1)NC(=O)[C@H](CCN)NC(=O)[C@H](CCNC(=O)[C@H](NC(=O)[C@H](CCN)NC(=O)[C@H](CCN)NC(=O)[C@H](CCN)NC(=O)Nc1ccccc1Cl)C(C)O)NC(=O)[C@H](CCN)NC(=O)[C@H](CCN)NC(=O)[C@H](C)O)C(=O)N[C@@H](CCN)Cc1ccccc1</smiles>

Figure 5 CB182 804

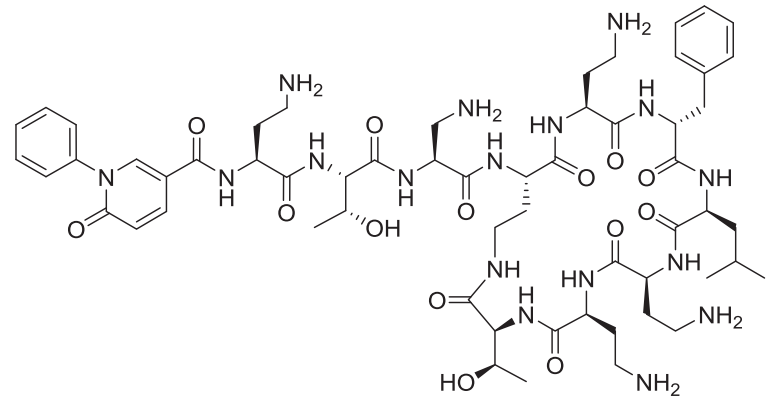

Figure 6 Pfizer $5 \times$.

An interesting further modification was the substitution of diaminobutyrate (Dab) at amino acid 3 by diaminopropionate (Dap). This substitution alone improved antibacterial activity whilst simultaneously decreasing the toxicity against a human proximal tubule cell line (hRPTEC). A 6-oxo-1-phenyl-1,6-dihydropyridine-3carbonyl derivative $(5 \times$; Figure 6$)$ was chosen for further study. $5 \times$ had $\mathrm{MIC}_{50}$ and $\mathrm{MIC}_{90}$ values against susceptible strains of $E$. coli, K. pneumoniae, $P$. aeruginosa and A. baumannii that were similar or twofold better than PMB. In a mouse thigh efficacy model, however, a $2-3 \times$ higher $f$ AUC/MIC was required to achieve the same reduction in bacterial counts as $\mathrm{PMB}$.

In terms of toxicity, $5 \times$ had a $\mathrm{TC}_{50}$ against the hRPTEC cell line of $>100 \mu \mathrm{M}$ compared with $22 \mu \mathrm{M}$ for PMB. Renal toxicity was investigated in both rat and dog. In rat there was evidence of improved safety compared with PMB. No histological lesions were noted after dosing $8 \mathrm{mg} \mathrm{kg}^{-1}$ per day for 7 days ( $f A U C 29 \mathrm{mg} \mathrm{h} \mathrm{ml}^{-1}$ ) whereas the same dose of PMB was not tolerated, and a lower dose of $4 \mathrm{mg} \mathrm{kg}^{-1}$ per day $\left(13 \mathrm{mg} \mathrm{h} \mathrm{ml}^{-1}\right)$ gave observable although minimal histopathological changes. In dog, however, results were more equivocal. The top dose of PMB $\left(6 \mathrm{mg} \mathrm{kg}^{-1}\right.$ per day; $\left.42 \mathrm{mg} \mathrm{h} \mathrm{ml}^{-1}\right)$ gave moderate lesions in two animals and marked lesions in a further 6 animals. A dose of $11 \mathrm{mg} \mathrm{kg}^{-1}$ per day $\left(64 \mathrm{mg} \mathrm{h} \mathrm{ml}^{-1}\right)$ of $5 \times$ gave moderate lesions in 5 animals and marked lesions in the other 3. The authors indicated that the overall therapeutic index did not appear to surpass that of $\mathrm{PMB}$.

\section{DIRECT ACTING DERIVATIVES WITH A REDUCED NUMBER OF POSITIVE CHARGES}

The concept that a reduced number of positive charges may lead to reduced toxicity has already been discussed in the context of the potentiator molecule NAB741. Northern Antibiotics has also developed direct acting compounds with a fatty acyl chain. A range of fatty acyl nonapeptide derivatives has been reported where amino acid 3 is

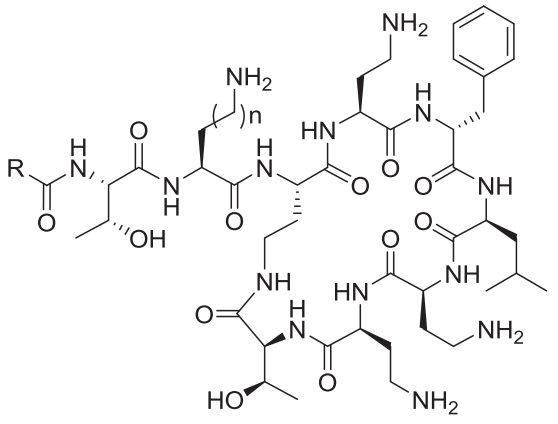

(a) CA824 $n=1, R=$<smiles>CCCN1CCNCC1CC(C)C</smiles>

(b) CA900 n=0, R=<smiles>CC(C)C[C@@H]1CNCC(C(C)C)C1</smiles>

(c) $\mathrm{CA} 1049 \mathrm{n}=0, \mathrm{R}=$<smiles>CCCCCCC(CN)C(C)C</smiles>

Figure 7 Cantab anti-infectives nonapeptides.

replaced with amino acids without an amine in the side chain thus resulting in molecules with only three positive charges. ${ }^{70,71}$ For optimal activity it appeared to be necessary to have a hydroxyl containing side chain, with D-serine or D-threonine apparently superior to L-serine. Compounds with aminobutyrate at amino acid 3, eg, NAB7061, had weak direct antimicrobial activity but retained the ability to potentiate other antibiotics such as rifampin. The acylated nonapeptide octanoyl-Thr-D-Ser-PMB ${ }^{3-10}$ (NAB739; Figure 4b) was selected for further evaluation.

NAB739 had $\mathrm{MIC}_{50}$ and $\mathrm{MIC}_{90}$ values within $2-4 \times$ that of $\mathrm{PMB}$ against enterobacteriaceae but activity was somewhat reduced against Acinetobacter spp. $(4 \times$ less potent than PMB $)$ and especially P. aeruginosa ( $\mathrm{MIC}_{90} 16 \mu \mathrm{g} \mathrm{ml}^{-1}$ cf $2 \mu \mathrm{g} \mathrm{ml}^{-1}$ for PMB). ${ }^{72} \mathrm{NAB} 739$ was somewhat less cytotoxic than PMB against both LLC-PK1 ${ }^{73}$ and HK-2 ( $\mathrm{IC}_{50} 337 \mu \mathrm{g} \mathrm{ml}^{-1}$ cf $13 \mu \mathrm{g} \mathrm{ml}^{-1}$ for $\left.\mathrm{PMB}\right)^{74}$ cell lines. The in vivo toxicity of NAB739 has not been reported, but interestingly the compound had $50 \times$ higher renal clearance than colistin in rats. ${ }^{75}$

\section{AMINOACYL NONAPEPTIDES}

Cantab anti-infectives reported a range of derivatives in which the $\mathrm{N}$-terminal acyl moiety together with amino acid 1 were replaced with an aminoacyl moiety. ${ }^{59}$ In some of the derivatives the amine moiety was cyclised into a piperidine or piperazine ring, and amino acid 3 was sometimes Dap rather than Dab as in Pfizer $5 \times$. Many derivatives had improved MIC profiles and reduced cytotoxicity (HK-2) compared with $\mathrm{PMB}$

The piperazine derivative CA824 (Figure 7a), piperidine CA900 (Figure 7b) and alkyl derivative CA1049 (Figure 7c) were investigated in further detail. ${ }^{76}$ Against E. coli, K. pneumoniae and P. aeruginosa the modal MIC values were similar or slightly improved compared with PMB. The derivatives differed more in activity against $A$. baumannii where CA824 and CA1049 were marginally inferior and superior to 


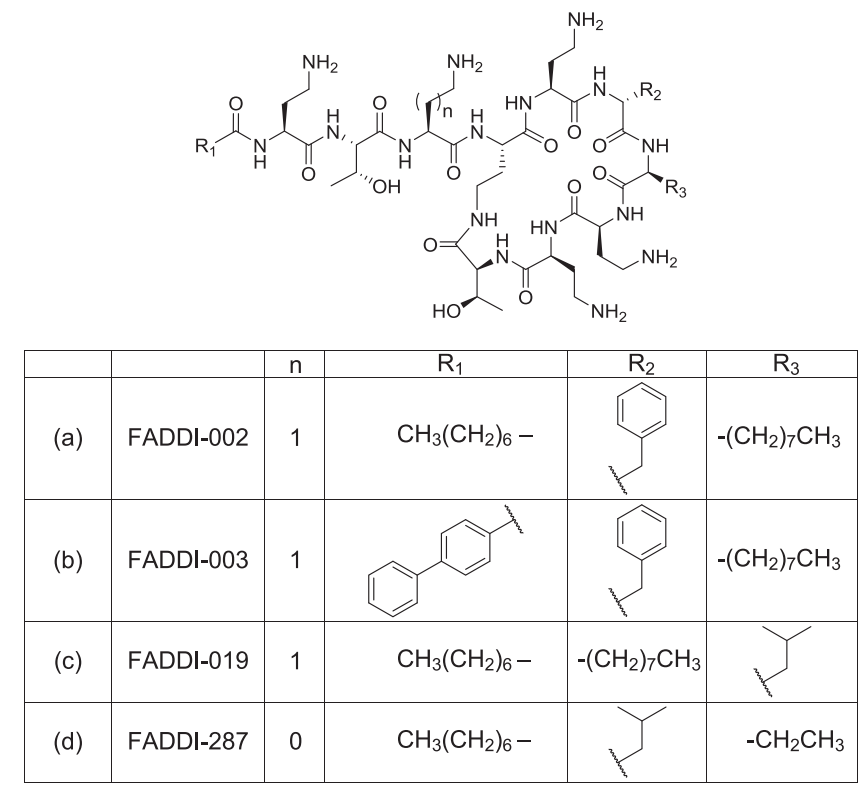

Figure 8 Monash decapeptides.

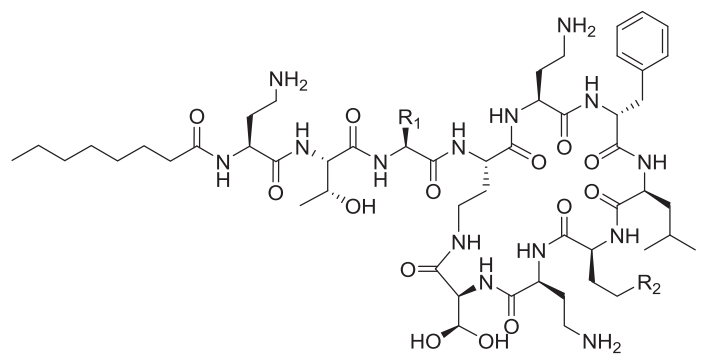

(a) $\mathrm{R}_{1}=\mathrm{CH}_{2} \mathrm{CH}_{2} \mathrm{NH}_{2}, \quad \mathrm{R}_{2}=\mathrm{CH}_{2} \mathrm{NHC}(=\mathrm{NH}) \mathrm{NH}_{2}$

(b) $\mathrm{R}_{1}=\mathrm{H} \quad \mathrm{R}_{2}=\mathrm{NH}_{2}$

Figure 9 University of Queensland decapeptides.
PMB, respectively, but CA900 was significantly improved with an $\mathrm{MIC}_{90}$ of $0.125 \mu \mathrm{g} \mathrm{ml}^{-1}$ compared to $1 \mu \mathrm{g} \mathrm{ml}^{-1}$ for PMB. All three derivatives had reduced cytotoxicity compared with $\mathrm{PMB}$ in the HK-2 cell line with $\mathrm{TC}_{50}$ values of $148 \mu \mathrm{g} \mathrm{ml}^{-1}$ (CA824), $167 \mu \mathrm{g} \mathrm{ml}^{-1}$ (CA900) and $64 \mu \mathrm{g} \mathrm{ml}^{-1}$ (CA1049) versus $15 \mu \mathrm{g} \mathrm{ml}^{-1}$ for PMB.

CA824 was progressed to in vivo efficacy studies. ${ }^{77}$ In a mouse thigh study, efficacy against $A$. baumannii was similar to PMB despite a $4 \times$ inferior MIC value. Efficacy was clearly superior to PMB in lung infection models against $A$. baumannii and $P$. aeruginosa.

\section{MONASH LIPOPEPTIDES}

Nation and co-workers ${ }^{45}$ have described a series of polymyxin lipopeptides specifically targeting polymyxin resistance, through the introduction of hydrophobic residues at positions 6 and/or 7 of the polymyxin core. Examples include FADDI-002 (L-octyl-glycine at position 7 with the $n$-octanoyl N-terminus), and FADDI-003 with the same core, but with the biphenylacyl N-terminus (Figure 8). These compounds show increased activity compared with PMB and Colistin against Polymyxin -resistant strains of Pseudomonas $\left(2-4 \mu \mathrm{g} \mathrm{ml}^{-1}\right.$ for FADDI-002 compared with 32->32 for PMB) and Acinetobacter (2-16 $\mu \mathrm{g} \mathrm{ml}^{-1}$ compared with 8-128 for PMB). Against polymyxinsensitive strains, however, the FADDI compounds were 1-2 dilutions less active in vitro. The increased activity against resistant strains was accompanied by increased activity against Gram-positive bacteria. The compounds showed high plasma protein binding as might be expected from their increased lipophilicity (97\% for FADDI-002 compared with $51 \%$ for PMB in human plasma). In rats, FADDI-002 and FADDI-003 showed lower clearance, and volume of distribution, and longer half-lives compared with Colistin.

FADDI-002 was taken into a lung infection model in the mouse with a polymyxin-resistant strain of Pseudomonas aeruginosa, where a significant reduction in bacterial counts was seen compared with control and to Colistin-treated animals (following a $40 \mathrm{mg} \mathrm{kg}^{-1}$ single dose FADDI-002 gave a $2.6 \log _{10}$ drop in bacterial counts $24 \mathrm{~h}$ post-infection, compared with non-treated control, whereas Colistin gave a $0.7 \log _{10}$ drop).

Table 3 Polymyxin analogues currently in development or which have contributed significantly to development of polymyxin SAR

\begin{tabular}{|c|c|c|}
\hline Compound & Figure & Comments \\
\hline PMBN & $3 b$ & $\begin{array}{l}\text { Inactive potentiator. } \mathrm{HK}-2 \mathrm{TC}_{50}>1000 \mu \mathrm{m} \text {. Reduced renal toxicity in rat model-no lesions at } 40 \mathrm{mg} \mathrm{kg}^{-1} \text { per day whereas PMB caused } \\
\text { significant lesions at } 25 \mathrm{mg} \mathrm{kg}^{-1} \text { per day }\end{array}$ \\
\hline [Dap1]-PMBN & $3 e$ & Pseudomonas-specific activity. Reduced toxicity in mouse- $\mathrm{LD}_{50} 23.5 \mu \mathrm{mol} \mathrm{kg}{ }^{-1}$ cf $4.8 \mu \mathrm{mol} \mathrm{kg}{ }^{-1}$ for PMB \\
\hline [Ser-3]-PMBO & $3 g$ & Pseudomonas-specific activity. Reduced toxicity in mouse- $\mathrm{LD}_{50}>50 \mu \mathrm{mol} \mathrm{kg}{ }^{-1}$ cf $4.8 \mu \mathrm{mol} \mathrm{kg}{ }^{-1}$ for PMB \\
\hline NAB741/SPR741 & $4 a$ & $\begin{array}{l}\text { Inactive potentiator. Reduced renal toxicity in Cynomolgus monkey model—no significant lesions at } 60 \mathrm{mg} \mathrm{kg}^{-1} \text { per day whereas PMB caused } \\
\text { lesions at } 12 \mathrm{mg} \mathrm{kg}^{-1} \text { per day. Inactive Pseudomonas }\end{array}$ \\
\hline NAB739 & $4 \mathrm{~b}$ & Activity similar to PMB except Pseudomonas. HK-2 $\mathrm{TC}_{50} 337 \mu \mathrm{g} \mathrm{ml}-1$ cf $13 \mu \mathrm{g} \mathrm{ml}^{-1}$ for PMB \\
\hline CB-182 804 & 5 & $\begin{array}{l}\text { Activity similar to PMB. LLC-PK1 } \mathrm{TC}_{50}>1000 \mu \mathrm{g} \mathrm{ml}-1 \text { cf } 318 \mu \mathrm{g} \mathrm{ml}-1 \text { for PMB. Reduced renal toxicity in Cynomolgus monkey-no lesions } \\
\text { at } 3.2 \mathrm{mg} \mathrm{kg}^{-1} \text { bid whereas PMB caused significant lesions at } 3.8 \mathrm{mg} \mathrm{kg}^{-1} \text { bid. Progressed to phase I clinical trial }\end{array}$ \\
\hline Pfizer $5 \times$ & 6 & $\begin{array}{l}\text { Activity similar to PMB. hRPTEC TC } 50>100 \mu \mathrm{m} \mathrm{cf} 22 \mu \mathrm{m} \text { for PMB. Reduced renal toxicity in rat and dog. Rat: no lesions at } 8 \mathrm{mg} \mathrm{kg}^{-1} \text { per day } \\
\text { whereas PMB caused significant lesions at } 4 \mathrm{mg} \mathrm{kg}^{-1} \text { per day. Dog: } 5 \text { animals moderate lesions and } 3 \text { marked at } 11 \mathrm{mg} \mathrm{kg}^{-1} \text { per day in } \\
\text { contrast to } 2 \text { moderate and } 6 \text { marked with PMB at } 6 \mathrm{mg} \mathrm{kg}^{-1} \text { per day }\end{array}$ \\
\hline CA824 & $7 a$ & Activity similar to PMB. HK-2 TC $50148 \mu \mathrm{g} \mathrm{ml} \mathrm{m}^{-1} \mathrm{cf} 15 \mu \mathrm{g} \mathrm{ml}-1$ for PMB. Superior efficacy to PMB in pneumonia models \\
\hline FADDI-02 & $8 a$ & Superior activity to PMB versus polymyxin-resistant strains \\
\hline FADDI-287 & $8 d$ & $\begin{array}{l}\text { Activity similar to PMB. Reduced renal toxicity in mouse. No lesions after } 6,10 \mathrm{mg} \mathrm{kg}^{-1} \text { doses whereas PMB caused moderate to severe } \\
\text { lesions with same dose regimen }\end{array}$ \\
\hline
\end{tabular}

Abbreviation: LD50, dose leading to death of 50\% of animals; PMB, polymyxin B; PMBN, polymyxin B nonapeptide; PMBO, polymyxin B octapeptide; TC50, concentration leading to 50\% reduction in cell viability. 
In terms of toxicity, FADDI-003 (8b), and a related analog, FADDI-019 (8c) were examined in a rat model of nephrotoxicity (sc injection over 3-4 days, accumulated dose $105 \mathrm{mg} \mathrm{kg}^{-1}$ ), and showed no histological findings (FADDI-002) or mild tubular dilation (FADDI-019). PMB treatment however, led to tubular damage and kidney lesions. The lower nephrotoxicity of the lipophilic compounds could be attributed to the higher protein binding, reducing the kidney exposure of the compounds.

The same group have recently filed a patent application on a series of less lipophilic polymyxin analogues ${ }^{78}$ in which core modifications particularly at positions 6 and 7 have been investigated. Some of these compounds have modifications such as substitution of Leu-7 with threonine, as found in Polymyxin M (vide infra). Many of the compounds show lower toxicity in vivo as judged in a mouse model of kidney injury (sc, $12 \mathrm{mg} \mathrm{kg}^{-1}, 6$ doses in 1 day). Histological examination revealed lower damage with the novel compounds compared to Polymyxin and Colistin.

Recently further data has been presented by Monash in conjunction with The Medicines Company on FADDI-287 ${ }^{79}$ which is a Polymyxin E derivative with aminobutyric acid rather than leucine at position 7 , together with Dap at position 3 (8d). FADDI-287 had similar in vitro activity to $\mathrm{PMB}$, promising efficacy in animal models of infection and showed reduced renal toxicity in a mouse repeat dose model showing no lesions after $6 \times 10 \mathrm{mg} \mathrm{kg}^{-1}$ doses. Under similar conditions all mice treated with $\mathrm{PMB}$ showed moderate to severe lesions.

\section{UNIVERSITY OF QUEENSLAND}

A recent publication by the University of Queensland ${ }^{51}$ has focussed on the reduction of nephrotoxicity, whilst also confirming some in vitro SAR trends within a series of decapeptides with an octanoyl amide at the N-terminus.

Within the heptapeptide ring, modification of the Dab residues by replacement with homoserine, $4^{\prime}$-amino phenylalanine, or acylation with arginine or glutamic acid gave variable results. No modification was tolerated at position 5 , moderate levels of activity were retained by modification at 9 , while position 8 was the most tolerant of modification. Replacement of Dab-8 with Arg-8 (compound 26, Figure 9a) led to in vitro activity comparable to PMB. Replacement of the amino acids at position 6 or 7 with lipophilic residues increased activity against resistant strains, but at the expense of cytotoxicity. Investigations into the linear peptide chain were also made, where replacement of Dab-3 with D-Dab or glycine were tolerated whereas replacement of Dab-1 with D-Dab led to significant loss of in vitro activity. In an assay using fresh primary human kidney cells, the gly-3 analog (compound 38; Figure $9 \mathrm{~b}$ ) demonstrated a reduction in release of lactate dehydrogenase and $\gamma$-glutamyl transferase compared with Colistin.

\section{CONCLUSIONS AND FUTURE DIRECTIONS}

Compared with well-established fields like the $\beta$-lactams, macrolides or even glycopeptides, development of next generation polymyxins is still in its infancy. Nevertheless, the tools available to assess renal toxicity including renal proximal tubule cell lines and sensitive urinary biomarkers of kidney injury for use in experimental animals, are allowing the SAR for toxicity to be investigated as well as that for activity. Further, advances in the basic scientific understanding of polymyxin toxicity will undoubtedly provide further insights into how to engineer less toxic molecules.

Progress is being made on multiple fronts. Potentiator compounds with reduced toxicity but limited direct activity, which potentiate the activity of other antibiotics, are perhaps currently most advanced. With regards to direct acting molecules with improved therapeutic index, CB-182 804 was progressed to human studies but discontinued. Nevertheless, there appear to be molecules with more promising profiles currently under evaluation. Finally, molecules such as FADDI-002 show that there is potential to introduce activity against polymyxin-resistant strains which may be of increasing importance given the emergence of the MCR-1 resistance mechanism. The properties of polymyxin analogues which are currently in development or which have contributed significantly to the understanding of polymyxin SAR are summarized in Table 3.

Given the dearth of alternative agents for MDR Gram-negative agents, it is clear that the polymyxins will have a role in this area for many years to come and next generation polymyxins may well have a major role to play in improving therapy beyond the current standard of care.

\section{CONFLICT OF INTEREST}

The authors are employees of Cantab Anti-infectives Ltd., which company has a commercial arrangement with Spero Therapeutics Ltd. Compounds under development by both companies are included in this review.

1 Ainsworth, G. C., Brown, A. M. \& Brownlee, G. Aerosporin, an antibiotic produced by Bacillus aerosporus Greer. Nature 159, 263 (1947).

2 Stansly, P. G., Shepherd, R. G. \& White, H. J. Polymyxin: a new chemotherapeutic agent. Bull. Johns Hopkins Hosp. 81, 43-54 (1947).

3 Schoenbach, E. B. \& Bryer, M. J. The clinical use of polymyxin. Bull. Johns Hopkins Hosp. 82, 637-639 (1948).

$4 \mathrm{Li}$, J. et al. Colistin: the re-emerging antibiotic for multi-drug resistant Gram-negative bacterial infections. Lancet Infect. Dis. 6, 589-601 (2006).

5 Landman, D., Georgescu, C., Martin, D. A. \& Quale, J. Polymyxins revisited. Clin. Microbiol. Rev. 21, 449-465 (2008).

6 Lim, L. M. et al. Resurgence of colistin: a review of resistance, toxicity, pharmacodynamics, and dosing. Pharmacotherapy 30, 1279-1291 (2010).

7 Liu, Y. Y. et al. Emergence of plasmid-mediated colistin resistance mechanism MCR-1 in animals and human beings in China: a microbiological and molecular biological study. Lancet Infect. Dis. 16, 161-168 (2016).

8 Sader, H. S., Farrell, D. J., Flamm, R. K. \& Jones, R. N. Antimicrobial susceptibility of gram-negative organisms isolated from patients hospitalized in intensive care units in United States and European Hospitals (2009-2011). Diagn. Microbiol. Infect. Dis. 78, 443-448 (2014).

9 Nation, R. L., Velkov, T. \& Li, J. Colistin and Polymyxin B: peas in a pod or chalk and cheese? Clin. Infect. Dis. 59, 88-94 (2014).

10 Elias, L. S., Konzen, D., Krebs, J. M. \& Zavascki, A. P. The impact of polymyxin B dosage on in-hospital mortality of patients treated with this antibiotic. J. Antimicrob. Chemother. 65, 2231-2237 (2010).

11 Nation, R. L. et al. Framework for optimisation of the clinical use of colistin and polymyxin B: the Prato polymyxin consensus. Lancet. Infect. Dis. 15, 225-234 (2015).

12 Rao, G. G. et al. New dosing strategies for an old antibiotic: pharmacodynamics of front-loaded regimens of colistin at simulated pharmacokinetics in patients with kidney or liver disease. Antimicrob. Agents Chemother. 58, 1381-1388 (2014).

13 Bergen, P. J. et al. Polymyxin combinations: pharmacokinetics and pharmacodynamics for rational use. Pharmacotherapy 35, 34-42 (2015).

14 Vaara, M. Polymyxins and their novel derivatives. Curr. Opin. Microbiol. 13, 574-581 (2010).

15 Vaara, M. Novel derivatives of polymyxins. J. Antimicrob. Chemother. 68, 1213-1219 (2013).

16 Velkov, T., Thompson, P. E., Nation, R. L. \& Li, J. Structure-activity relationships of polymyxin antibiotics. J. Med. Chem. 53, 1898-1916 (2010).

17 Velkov, T., Roberts, K. D., Nation, R. L., Thompson, P. E. \& Li, J. Pharmacology of polymyxins: new insights into an "old" class of antibiotics. Future Microbiol. 8(6), 711-724 (2013).

18 Kadar, B., Kocis, B., Nagy, K. \& Szabo, D. The Renaissance of polymyxins. Curr. Med. Chem. 20, 3759-3773 (2013).

19 Orwa, J. A. et al. Isolation and structural characterisation of Polymyxin B components. J. Chromatogr. A 912, 369-373 (2001).

20 Kline, T., Holub, D., Therrien, J., Leung, T. \& Ryckman, D. Synthesis and characterisation of the colistin peptide polymyxin E1 and related peptides. J. Peptide Res. 57 175-187 (2001).

21 Shoji, J., Kato, T. \& Hinoo, H. The structure of polymyxin S. (Studies on antibiotics from the genus Bacillus. XXI). J. Antibiot. 30, 1035-1041 (1977).

22 Martin, N. I. et al. Isolation, structural characterisation and properties of Mattacin (Polymyxin M), a cyclic peptide antibiotic produced by Paenobacillus kobensis $\mathrm{M}$. J. Biol. Chem. 278(15), 13124-13132 (2003). 
23 Shaheen, M., Li, J., Ross, A. C., Vederas, J. C. \& Jensen, S. E. Paenibacillus polymyxa PKB1 produces variants of Polymyxin B-Type Antibiotics. Chem. Biol. 18, 1640-1648 (2011).

24 Shoji, J., Kato, T. \& Hinoo, H. The structure of Polymyxin T1 (Studies on antibiotics from the genus Bacillus XXII). J. Antibiot. 30(12), 1042-1048 (1977).

25 Storm, D. R., Rosenthal, K. S. \& Swanson, P. E. Polymyxin and related peptide antibiotics. Ann. Rev. Biochem. 46, 723-763 (1977).

26 Rosenthal, K. S., Ferguson, R. A. \& Storm, D. R. Mechanism of action of EM49, membrane-active peptide antibiotic. Antimicrob. Agents Chemother. 12(6), 665-672 (1977).

27 Parker, W. L. \& Rathnum, M. L. EM49, a new peptide antibiotic IV. The structure of EM49. J. Antibiot. 28, 379-389 (1975).

28 Shoji, J. et al. Isolation of a new antibiotic 333-25, related to antibiotic EM 49. (Studies on antibiotics from the genus Bacillus. XI). J. Antibiot. 29, 516-520 (1976).

29 Sugawara, K. et al. Bu-2470, a new peptide antibiotic complex. II. Structure determination of Bu-2470A, B1, B2a and B2b. J. Antibiot. 36(6), 634-638 (1983).

30 Kato, T. \& Shoji, J. The structure of octapeptin D (studies on antibiotics from the genus Bacillus. XXVIII). J. Antibiot. 33(2), 186-191 (1980).

31 Qian, C.-D. et al. Battacin (Octapeptin B5), a new cyclic lipopeptide antibiotic from paenibacillus tianmuensis active against multidrug-resistant gram-negative bacteria. Antimicrob. Agents Chemother. 56(3), 1458-1465 (2012).

32 De Zoysa, G. H., Cameron, A. J., Hegde, V. V., Raghothama, S. \& Saronji, V. Antimicrobial peptides with potential for biofilm eradication: synthesis and structure activity relationship studies of Battacin Peptides. J. Med. Chem. 58, 625-639 (2015)

33 Mares, J., Kumaran, S., Gobbo, M. \& Zerbe, O. Interactions of lipopolyssaccharide and polymyxin studied by nmr spectroscopy. J. Biol. Chem. 284, 11498-11506 (2009).

34 Vaara, M. Agents that increase the permeability of the outer membrane. Microbiol. Rev. 56, 395-411 (1992).

35 Deris, Z. Z. et al. Probing the penetration of antimicrobial polymyxin lipopeptides into Gram-negative bacteria. Bioconjug. Chem. 25, 750-760 (2014).

36 Kelesidis, T. \& Falagas, M. E. The safety of polymyxin antibiotics. Exp. Opin. Drug Safety 14, 1687-1701 (2015).

37 Justo, J. A. \& Bosso, J. A. Adverse reactions associated with systemic polymyxin therapy. Pharmacotherapy 35, 28-33 (2015).

38 Phe, K. et al. In vitro assessment and multi center color study of comparative nephrotoxicity rates associated with colistimethate versus polymyxin B therapy. Antimicrob. Agents Chemother. 58, 2740-2746 (2014).

39 Rigatto, M. H. et al. Multicenter prospective color study of renal failure in patients treated with colistin versus polymyxin B. Antimicrob. Agents Chemother. 60 2443-2449 (2016)

40 Azad, M. A. K., Akter, J., Rogers, K. L., Nation, R. L. \& Li, J. Major pathways of polymyxin-induced apoptosis in rat kidney proximal tubular cells. Antimicrob. Agents Chemother. 59, 2136-2143 (2015).

41 Vaara, M. et al. Novel Polymyxin derivatives carrying only three positive charges are effective antibacterial agents. Antimicrob. Agents Chemother. 52(9), 3229-3236 (2008).

42 Huang, J. X. et al. Evaluation of biomarkers for in vitro prediction of drug-induced nephrotoxicity; comparison of HK-2, immortalized human proximal tubule epithelial, and primary cultures of human proximal tubular cells. Pharmacol. Res. Perspect. 3, e00148 (2015)

43 Olaitan, A. O., Morand, S. \& Rolain, J. M. Mechanisms of polymyxin resistance: acquired and intrinsic resistance in bacteria. Front. Microbiol. 5, 643 (2014).

44 Sharma, S. K. et al. Solid-Phase total synthesis of Polymyxin B1. J. Pept. Res. 53, 501-506 (1999)

45 Velkov, T. et al. Teaching 'old' Polymyxins new tricks: new-generation lipopeptides targeting Gram-negative 'superbugs'. ACS Chem. Biol. 9(5), 1172-1177 (2014).

46 Kanazawa, K. et al. Contribution of each amino acid residue in Polymyxin $B_{3}$ to antimicrobial and lipopolysaccharide binding activity. Chem. Pharm. Bull. 57, 240-244 (2009).

47 Ramesh, S., Govender, T., Kruger, H. G., Alberico, F. \& de la Torre, B. G. An improved and efficient strategy for the total synthesis of a colistin-like peptide. Tetrahedron Lett. 57, 1885-1888 (2016)

48 De Visser, P. C. et al. Solid-phase synthesis of polymyxin B1 and analogues via a safetycatch approach. J. Pept. Res. 61, 298-306 (2003).

49 Magee, T. V. et al. Discovery of Dap-3 polymyxin analogues for the treatment of multidrug-resistant Gram-negative nosocomial infections. J. Med. Chem. 56, 5079-5093 (2013)

$50 \mathrm{Xu}, \mathrm{W} .-\mathrm{L}$. et al. A new strategy for the total solid-phase synthesis of polymyxins. Tetrahedron Lett. 56, 4796-4799 (2015).
51 Gallardo-Godoy, A. et al. Activity and predicted nephrotoxicity of Synthetic Antibiotics based on Polymyxin B. J. Med. Chem. 59(3), 1068-1077 (2016).

52 Weinstein, J., Afonso, A., Moss, E. \& Miller, G. Selective chemical modifications of Polymyxin B. BioOrg. Med. Chem. Lett. 8, 3391-3396 (1998).

53 Kimura, Y., Matsunaga, H. \& Vaara, M. Polymyxin B octapeptide and Polymyxin B heptapeptide are potent outer membrane permeability-increasing agents. J. Antibiot. 45, 742-749 (1992)

54 Okimura, K., Ohki, K., Sato, Y., Ohnishi, K. \& Sakura, N. Semi-synthesis of Polymyxin B (2-10) and Colistin (2-10) analogs employing the Trichloroethoxycarbonyl (Troc) group for side chain protection of $\alpha, \gamma$-diaminobutyric acid residues. Chem. Pharm. Bull. 55 (12), 1724-1730 (2007)

55 O'Dowd, H. et al. Preparation of tetra-BOC protected polymyxin B nonapeptide. Tetrahedron Lett. 48, 2003-2005 (2007).

56 Leese, R. A. (BioSource Pharm. Inc.) Antibiotic compositions for the treatment of Gram Negative Infections. WO2010075416 (2010).

57 Fauchere, J. L. \& Mosbach, K. Production of Covalently-linked Conjugates from an Antibiotic and a Non-toxic derivative of Polymyxin B. W08800950 (1988).

58 Chen, J. M., Li, Z. B., Magee, T. V. \& Martinez, C. A. (Pfizer Inc.) Polymyxin derivatives useful as antibacterial agents. WO2012168820 (2012).

59 Brown, P. et al. Poster F-739, 55th Interscience Conference on Antimicrobial Agents and Chemotherapy (San Diego, CA, USA, 2015)

60 Vaara, M. \& Vaara, T. Sensitization of Gram-negative bacteria to antibiotics and complement by a nontoxic oligopeptide. Nature 303, 526-528 (1983).

61 Keirstead, N. D. et al. Early prediction of polymyxin-induced nephrotoxicity with next-generation urinary kidney injury biomarkers. Toxicol. Sci. 137, 278-291 (2014).

62 Vaara, M. et al. A novel Polymyxin derivative that lacks the fatty acyl tail and carries only three positive charges has strong synergism with agents excluded by the intact outer membrane. Antimicrob. Agents Chemother. 54, 3341-3346 (2010).

63 Okimura, K. et al. Chemical conversion of natural polymyxin B and colistin to their N-terminal derivatives. Bull. Chem. Soc. Jpn. 80, 543-552 (2007).

64 Coleman, S., Bleavins, M., Lister, T., Vaara, M. \& Parr, T. J. The Assessment of SPR741 for Nephrotoxicity in Cynomolgus Monkeys and Sprague-Dawley rats. Poster Monday523 (ASM Microbe, Boston, MA, USA, 2016).

65 Katsuma, N. et al. Development of des-fatty acyl-Polymyxin B decapeptide analogs with Pseudomonas aeruginosa-specific antimicrobial activity. Chem. Pharm. Bull. 57, 332-336 (2009).

66 Sato, Y., Shindo, M., Sakura, N., Uchida, Y. \& Kato, I. Novel des-fatty acyl Polymyxin B derivatives with Pseudomonas aeruginosa-specific antimicrobial activity. Chem. Pharm. Bull. 59, 597-602 (2011).

67 Quale, J. et al. Activity of Polymyxin B and the novel polymyxin analogue CB-182,804 against contemporary Gram-negative pathogens in New York City. Microb. Drug Resist. 18, 132-136 (2012)

68 Arya, A. et al. Poster F1-1627, 50th Intersci. Conf. Antimicrob. Agents Chemother. (Boston, USA, 2010).

69 Coleman, S., Deats, T., Pawliuk, R., Chavan, A. \& Oleson, F. Poster F-1630, 50th Interscience Conference on Antimicrobial Agents and Chemotherapy (Boston, MA, USA, 2010).

70 Vaara, $M$. et al. Novel polymyxin derivatives carrying only three positive charges are effective antibacterial agents. Antimicrob. Agents Chemother. 52, 3229-3236 (2008).

71 Vaara, M. \& Vaara, T. Structure-activity studies on novel polymyxin derivatives that carry only three positive charges. Peptides 31, 2318-2321 (2010).

72 Vaara, M., Sader, H. S., Rhomberh, P. R., Jones, R. N. \& Vaara, T. Antimicrobial activity of the novel polymyxin derivative NAB739 tested against Gram-negative pathogens. J. Antimicrob. Chemother. 68, 636-639 (2013).

73 Mingeot-Leclerq, M. P., Tulkens, P. M., Denamur, S., Vaara, T. \& Vaara, M. Novel polymyxin derivatives are less cytotoxic than polymyxin $B$ to renal proximal tubular cells. Peptides 35, 248-252 (2012).

74 Vaara, M. \& Vaara, T. The novel polymyxin derivative NAB739 is remarkably less cytotoxic than polymyxin B and colistin to human kidney proximal tubule cells. Int. J. Antimicrob. Agents 41, 292-293 (2013).

75 Ali, F. E. A. et al. Pharmacokinetics of novel antimicrobial cationic peptides NAB7061 and NAB739 in rats following intravenous administration. J. Antimicrob. Chemother. 64, 1067-1070 (2009)

76 Wiederhold, N. P. et al. Poster F-734, 55th Interscience Conference on Antimicrobial Agents and Chemotherapy (San Diego, CA, USA, 2015).

77 Boakes, S. et al. Poster F-735, 55th Interscience Conference on Antimicrobial Agents and Chemotherapy (San Diego, CA, USA, 2015).

$78 \mathrm{Li}$, J., Nation, R., Velkov, T., Thompson, P. \& Roberts, K. D. (Monash University). Polymyxin derivatives as antimicrobial compounds. W02015149131 (2015).

79 Sabet et al. Pharmacology of the Novel Polymyxin Faddi-287 in Preclinical Models. Poster Saturday-499 (ASM Microbe, Boston, MA, USA, 2016). 